\title{
The Validity of Balance Evaluation of Patients with Orthopaedic Knee Problems using Nintendo Wii ${ }^{\circledR}$
}

\author{
Cihan $\mathrm{CA}^{1 *}$, Ummuhan $\mathrm{BA}^{2}$, Ferruh $\mathrm{T}^{1}$ and \\ Sermet $\mathbf{I}^{3}$ \\ ${ }^{1}$ Department of Physiotherapy and Rehabilitation, Schoo \\ of Health Sciences, Dumlupinar University, Evliya Celebi \\ Campus, 43444, Kutahya, Turkey \\ ${ }^{2}$ School of Physiotherapy and Rehabilitation, Pamukkale \\ University, Kınıklı Campus, 20070, Denizli, Turkey \\ ${ }^{3}$ Department of Orthopedics and Traumatology, Faculty \\ of Medicine, Dumlupinar University, Evliya Celebi \\ Campus, 43444, Kutahya, Turkey \\ *Corresponding author: Cihan CA, Department of \\ Physiotherapy and Rehabilitation, School of Health \\ Sciences, Dumlupinar University, 43444, Kutahya, \\ Turkey
}

Received: March 06, 2017; Accepted: March 29, 2017; Published: April 04, 2017

\begin{abstract}
Objectives: In recent years, the Nintendo $\mathrm{Wii}^{\circledR}(\mathrm{NW})$ has been widely used in the field of healthcare. There are studies in literature which have used the closed source encoded software of NW in the evaluation of balance. However, the availability of this software is limited in clinical practice. Therefore, in this study it was aimed to examine the relationship between the results of NW balance evaluation made without closed source encoded software with the results of the Kinesthetic Ability Trainer $^{\circledast}$ (KAT) and the Single Leg Stance Test (SLST).
\end{abstract}

Materials and Methods: The study included 33 volunteers aged $23.85 \pm 4.62$ years with a chronic orthopaedic knee joint pathology. The balance of the participants was evaluated with NW, KAT and SLST.

Results: A significant correlation at a low level was seen in only 1 parameter between the balance results of NW and KAT $(p<0.05)$. No signficant correlation was observed in the other parameters. A significant relationship was determined between the double foot NW area, the mediolateral (ML) width, affected side ML width and weight-bearing asymmetry (WBA) parameters and the SLST results $(p<0.05)$ at a weak-moderate level $(r=-0.38, r=-0.50, r=-0.36, r=-0.38)$.

Conclusion: In conclusion, NW can be used instead of subjectve tests such as SLST. However, KAT uses a complex evaluation algorithm and provides more sensitive results. Therefore, to be able to increase the sensitivity of the NW balance evaluation system, standardised evaluation procedures should be developed.

Keywords: Virtual reality; Balance; Knee injury; Orthopaedic

\section{Introduction}

Balance is defined as the ability to hold the centre of body weight within a support surface $[1,2]$. There are various methods which can be used in the evaluation of balance. Each of these evaluates balance at a different level [3]. The localisation of the centre of weight varies in different patient groups (lower extremity injuries, osteoarthritis, patients with total knee prosthesis, stroke etc). This leads to weightbearing asymmetry (WBA) [4]. In the evaluation of balance and WBA, force platforms are accepted as the gold standard. The applicability of these in clinics is limited as they are expensive and are difficult to transport from one place to another and then set up. However, as a low-cost alternative, virtual reality systems may provide rapid, easy, valid and reliable data in the evaluation of both balance and WBA [5-10].

The Nintendo Wii ${ }^{\oplus}$ (NW) game console is readily available compared to laboratory-based virtual reality systems. On the Wii Balance Board, which is an accessory of NW, there are 4 sensors. Weight distribution and changes in the pressure centre are determined through these sensors [11-15]. Compared to other evaluation devices, the low cost and portable structure are the greatest advantages $[6,16-$ 19]. Studies which have been conducted on balance evaluation with NW have generally used closed source encoded software. Due to the difficulty of the availability of this software, the utility of NW in clinical practice is limited. Therefore, in this study it was aimed to examine the relationship between the results of NW balance evaluation made without closed source encoded software with the results of the Kinesthetic Ability Trainer (KAT) and the Single Leg Stance Test (SLST).

\section{Materials and Methods}

This study was conducted on volunteer patients in the Physiotherapy and Rehabilitation Department of Dumlupinar University, School of Health Science, between June 2015 and December 2015. Informed consent was obtained from all the participants before starting the study.

The study enrolled a total of 40 cases diagnosed with a chronic knee joint problem by an Orthopaedics and Traumatology specialist (SI). A total of 7 patients were excluded as 5 did not wish to participate and 2 had additional health problems. Thus the study was completed with 33 participants with a mean age of $23.85 \pm 4.62$ years. Approval for the study was granted by Pamukkale University Non-Interventional Clinical Research Ethics Committee. The inclusion and exclusion criteria are presented below:

Study inclusion criteria:

- Having an orthopaedic knee joint pathology for at least 6 months
Phys Med Rehabil Int - Volume 4 Issue 1 - 2017 ISSN : 2471-0377 | www.austinpublishing group.com Cihan et al. @ All rights are reserved 


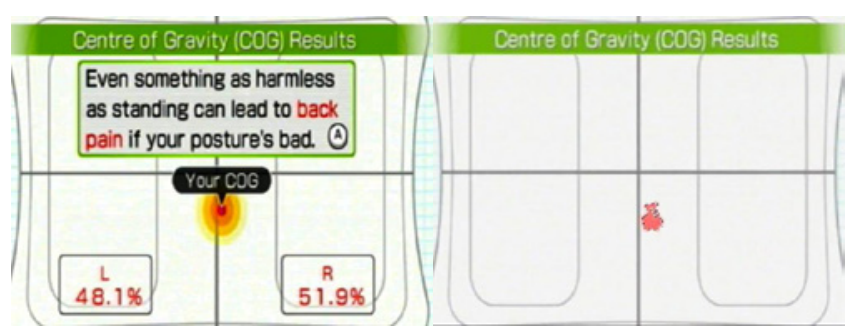

Figure 1: Screenshots of weight bearing asymmetry evaluation and area with NW

\section{Aged 19-40 years}

- Ambulatory without the use of any assistive device (crutches, cane etc)

Study exclusion criteria:

- $\quad$ Having an acute phase knee pathology

- $\quad$ Receiving treatment for the knee problem

- $\quad$ Having undergone knee surgery

- Having another health problem which could affect balance

\section{Evaluation}

The evaluations were made a single researcher (CCA). Sociodemographic information and data of the pathologies of the participants were recorded in face-to-face interviews. A Visual Analog Scale (VAS) was used to assess the severity of pain. All the data were gathered under the same environmental conditions (fixed lighting, temperature, etc). The evaluations were repeated 3 times on both feet and on the affected side. For the balance evaluations, the participants were requested to step on to the platform for each repetition with the same foot and measurements were made without shoes and wearing normal daytime clothes. Using the reference points on the KAT and the Wii Balance Board, the same placements of the feet were achieved. The balance evaluation methods researched in the study are explained below.

\section{Balance evaluations}

Nintendo Wii $^{\oplus}$ Balance Evaluations: Calibration was made before the evaluations. The oscillations of the pressure centre and the WBA were evaluated with the Wii Fit ${ }^{\text {tix }}$ balance tests. During the test, the participants were requested to maintain balance for as long as possible while looking at a red point $10 \mathrm{~cm}$ in diameter at a distance of $1.5 \mathrm{~m}$. The screen images of the evaluations were recorded by transfer to the computer with the installation of EasyCAP ${ }^{\mathrm{m} w}$, RCA cable and connectors. These images were standardised with Adobe Photoshop $\mathrm{CS}^{\circ}$. The parameters related to the pressure centre oscillations were counted 3 times as pixels using the rapid selection tool and were recorded (Figure 1). The mean of these 3 measurements was used in the analyses.

Kinesthetic ability trainer ${ }^{\circledR}$ balance evaluations: The balance of participants was evaluated with KAT (Ts650, SportKAT LLC, USA). The procedure defined by the manufacturer was used for the evaluation.
Table 1: Characteristics of the patients.

\begin{tabular}{|c|c|c|c|}
\hline Variables & $\mathbf{n}$ & $\mathbf{X} \pm$ SD & min-max \\
\hline Age (Year) & 33 & $23.85 \pm 4.6$ & $19-36$ \\
\hline Height (cm) & 33 & $174.03 \pm 7.7$ & $156-190$ \\
\hline Weight (kg) & 33 & $72.15 \pm 16.6$ & $45-110$ \\
\hline BMI (kg/m²) & 33 & $23.55 \pm 3.7$ & $17.7-30.5$ \\
\hline Rest Pain (VAS) & 33 & $1.52 \pm 1.9$ & $0-6.4$ \\
\hline $\begin{array}{c}\text { Activity Pain } \\
\text { (VAS) }\end{array}$ & 33 & $2.53 \pm 1.8$ & $0-6.9$ \\
\hline $\begin{array}{c}\text { Night Pain (VAS) } \\
\text { Disease Duration } \\
\text { (Month) }\end{array}$ & 33 & $1.97 \pm 2.9$ & $0-58.5$ \\
\hline BMI: Body Mass & 33 & $32.91 \pm 24.2$ & $9-108$ \\
\hline
\end{tabular}

BMI: Body Mass Index; cm: centimeter; kg: kilogram; m: meter; max: maximum min: minimum; n: number of case; SD: Standard Deviation; VAS: Visually Analog Scale; $X:$ average.

Table 2: Descriptive values of the patiens.

\begin{tabular}{|c|c|c|}
\hline Variables & $\mathbf{n}$ & $\%$ \\
\hline \multicolumn{3}{|l|}{ Gender } \\
\hline Male & 21 & 63.6 \\
\hline Female & 12 & 34.6 \\
\hline \multicolumn{3}{|c|}{ Dominant Lower Extremity } \\
\hline Right & 26 & 78.8 \\
\hline Left & 7 & 21.2 \\
\hline \multicolumn{3}{|l|}{ Diagnosis } \\
\hline Meniscopathy & 9 & 27.3 \\
\hline Patellar Tendinitis & 6 & 18.1 \\
\hline PPS & 16 & 48.5 \\
\hline ACL Injury & 2 & 6.1 \\
\hline \multicolumn{3}{|l|}{ Affected Side } \\
\hline Right & 17 & 51.5 \\
\hline Left & 16 & 48.5 \\
\hline
\end{tabular}

ACL: Anterior Cruciate Ligament; $n$ : number of case; PPS: Patellofemoral Pain Syndrome; \%: percent.

Calibration of the platform was made before each measurement. The balance result, ML and AP rates obtained from the measurements were recorded. After a review of the consistency of the measurements in the manner recommended by the manufacturer, the best result was used in the analyses.

Single leg stance test: For the evaluation of static balance, the SLST was also used. In this test, the participants were requested to cross their hands across their shoulders and stand with the knee of the unaffected side in $90^{\circ}$ flexion and hold this posture while looking at a red point $10 \mathrm{~cm}$ in diameter at a distance of $1.5 \mathrm{~m}$. A chronometer was started at the moment the foot was raised from the floor and the period was recorded until the raised foot made contact with the floor and the position of the weight-bearing foot was changed [20,21].

\section{Statistical analysis}

The data obtained in the study were analysed using SPSS 20.0 statistics software. Descriptive data were stated as mean, frequency, minimum-maximum, standard deviation and percentage values. The data were analysed with the Spearman test. A value of $p<0.05$ was accepted as statistically significant. 


\section{Results}

The mean duration of disease was $32.9 \pm 24.2$ months. The values related to age, height, body weight, body mass index, and pain levels at rest, in activity and at night are shown in Table 1 and descriptive data in Table 2.

The relationship was examined between the NW, KAT and SLST parameters on both feet. No relationship was observed between NW and KAT parameters. A significant correlation was determined between the NW area and the SLST parameter at a weak level ( $r=-$ 0.38). A significant relationship was observed at a moderate level between the ML width parameter and the SLST results $(r=-0.50)$. As a result, when the area of the NW and ML width parameters decreased, the time standing on one foot increased. The data ofthese results are shown in Table 3.

The relationship was examined of the NW, KAT and SLST parameters of the affected side. A significant correlation at a weak level was observed between the AP width of NW and the KAT balance parameter $(\mathrm{r}=0.39)$. A significant correlation at a weak level was observed between the ML width parameter of NW and SLST ( $r=-$ 0.36). A significant correlation at a weak level was observed between the WBA parameter of NW and SLST $(r=-0.38)$. No significant relationship was determined between the other parameters. The data of these findings are shown in Table 4.

\section{Discussion}

As a result of the study evaluations, while there was a correlation between NW and KAT in only 1 parameter, there was seen to be a correlation in several parameters between NW and SLST. Therefore, NW can be used instead of subjective tests such as SLST. However, systems such as KAT use complex evaluation algorithms, so to be able to use NW for balance evaluation in place of systems such as KAT, the sensitivity must be increased and standardised evaluation procedures must be developed.

There is a current need for easily accessible, valid and reliable balance evaluation systems. NW without closed source encoded software is a method which is readily available with its own evaluation Table 3: Investigation of the relationship between double-leg KAT, NW and affected side SLST of the cases.

\begin{tabular}{|c|c|c|c|c|c|c|}
\hline \multirow{2}{*}{\multicolumn{3}{|c|}{ Variables }} & \multicolumn{3}{|c|}{ Double-leg KAT $(n=33)$} & \multirow{3}{*}{$\begin{array}{l}\text { SLST } \\
-0.38\end{array}$} \\
\hline & & & \multirow{2}{*}{$\begin{array}{c}\text { Balance score } \\
0.07\end{array}$} & \multirow{2}{*}{$\begin{array}{c}\text { ML ratio } \\
0.16\end{array}$} & \multirow{2}{*}{\begin{tabular}{|c|} 
AP ratio \\
0.24
\end{tabular}} & \\
\hline \multirow{8}{*}{ 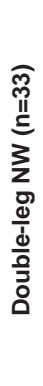 } & \multirow{2}{*}{ Area } & $r$ & & & & \\
\hline & & p & 0.71 & 0.38 & 0.18 & 0.03 \\
\hline & \multirow{2}{*}{ ML width } & $r$ & 0.05 & -0.12 & -0.19 & -0.50 \\
\hline & & p & 0.77 & 0.52 & 0.27 & 0.00 \\
\hline & \multirow{2}{*}{ AP width } & $r$ & 0.27 & 0.04 & -0.07 & -0.22 \\
\hline & & p & 0.13 & 0.83 & 0.71 & 0.21 \\
\hline & \multirow{2}{*}{ Weight Bearing Ratio } & $r$ & 0.24 & -0.07 & -0.03 & 0.09 \\
\hline & & p & 0.18 & 0.72 & 0.87 & 0.60 \\
\hline \multirow{2}{*}{\multicolumn{2}{|c|}{ SLST }} & $r$ & 0.12 & 0.01 & 0.07 & 1 \\
\hline & & p & 0.50 & 0.94 & 0.71 & 1 \\
\hline
\end{tabular}

AP: Anterioposterior; KAT: Kinesthetic Ability Trainer ${ }^{\circledR}$; ML: Mediolateral; $n$ : number of case; NW: Nintendo Wii ${ }^{-}$; r: Spearman Correlation Coefficient; p: level of significance; SLST: Single Leg Stance Test.
Table 4: Examination of the relationship between affected side KAT, NW and SLST of cases.

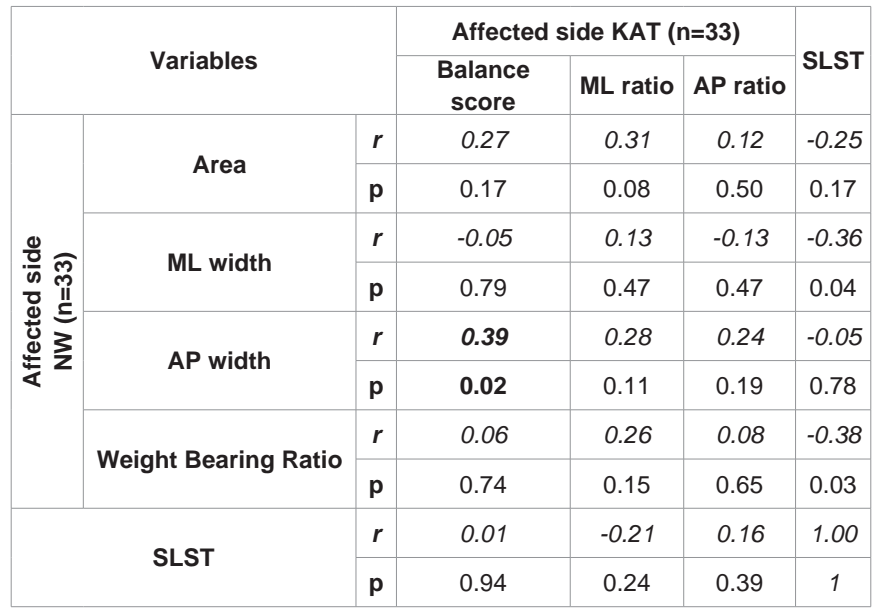

AP: Anterioposterior, KAT: Kinesthetic Ability Trainer ${ }^{\circledR}$; ML: Mediolateral; $n$ : number of case; NW: Nintendo Wii ${ }^{\circledR}$; r: Spearman Correlation Coefficient; $p$ : level of significance; SLST: Single Leg Stance Test.

results. However, few studies have examined the evaluation results of NW. In a study of healthy indviduals by Deans in 2011, the screen images of the NW balance test were placed on a co-ordinated plane [22]. From there, the data obtained were compared with the force platform and Biodex Balance System results. With the force platform results, a relationship was seen only with the ML width parameter. With the Biodex Balance System, a correlation was reported with the $\mathrm{AP}$ and ML width parameters of NW. To the best of our knowledge, there is no study in literature which has used the data from the Wii Balance Board transmitted to a computer rather than the data of the NW system with closed source encoded software.

In studies conducted on healthy individuals, patients diagnosed with Parkinson's disease and children with neurological problems, high consistency was revealed between Wii Balance Board balance evaluatons and force platform evaluations $[6,11,14,23-26]$. In the WBA evaluation in studies which used similar software in healthy indvduals and patients who had undergone hip or knee arthroplasty, consistency was reported between the Wii Balance Board and force platforms $[8,27]$. In those studies, only the data obtained from the Wii Balance Board were used instead of the information from the NW system. The development of this software is not possible in every clinic or research centre.

To the best of our knowledge, there is no study which has examined the correlation between NW and SLST. In the current study, a weak-moderate level of consistency was observed between the two balance evaluations. The balance evaluation systems of the NW and SLST are based on similar parameters. In both tests the main parameter is defined as the loss of balance while standing on one foot.

The incompatability between NW and KAT revealed by the results of this study is thought to arise for different reasons. First, the sensitivity of NW may be less than that of the force platform. In a study by Bartlett et al (2014) the force platform was reported to be approximately 8-fold more sensitive than NW [28]. This finding supports the opinions of the current study. Second, there is no standardised evaluation procedure for NW as there is in the other balance evaluation methods used in the current study. NW and KAT 
use different evaluation algorithms. In KAT, evaluation is made on a pneumatic base and in NW, the base is fixed and hard. This could be a reason for the low consistency seen. Jorgensen et al (2014) reported a correlation between parameters obtained from the fixed force platform and the NW Stillness Test results showing oscillations of the pressure centre during static standing, which supports the findings of the current study [29]. Finally, these results may have been due to the inclusion in the study of participants who were young, had low levels of pain and long-term disorders. Greater balance problems may be seen in those with more serious orthopaedic problems and high levels of pain and the NW evaluation results of these groups may show a higher level of correlation with other systems.

\section{Conclusion}

The results obtained in this study showed no correlation between the KAT balance evaluation system and the NW without closed source encoded software. However, there was a correlation in some parameters between the NW results and the results of SLST, which is widely used in balance evaluation in clinics and for which validity has been proven. Although the data obtained from the NW system is not as sensitive as KAT, they show a similar sensitivity to those of SLST, which is used in clinics. To be able to increase this sensitivity, there is a need to establish a standardised evaluation procedure.

\section{Acknowledgement}

This study was supported by Pamukkale University Scientific Research Projects Co-ordination Unit (project no.2013SBE013).

\section{References}

1. Hansen MS, Dieckmann B, Jensen K, Jakobsen BW. The reliability of balance tests performed on the kinesthetic ability trainer (KAT 2000). Knee Surg Sports Traumatol Arthrosc. 2000; 8: 180-185.

2. Hrysomallis C. Relationship between balance ability, training and sports injury risk. Sport Med. 2007; 37: 547-556.

3. Panjan A, Sarabon N. Review of Methods for the Evaluation of Human Body Balance. Sport Sci Rev. 2010; 19: 131-163.

4. Kumar SNS, Omar B, Joseph LH, Hamdan N, Htwe O, Hamidun N. Accuracy of a Digital Weight Scale Relative to the Nintendo Wii in Measuring Limb Load Asymmetry. J Phys Ther Sci. 2014; 26: 1205-1207.

5. Salavati M, Hadian MR, Mazaheri M, Negahban H, Ebrahimi I, Talebian S et al. Test-retest reliabty of center of pressure measures of postural stability during quiet standing in a group with musculoskeletal disorders consisting of low back pain, anterior cruciate ligament injury and functional ankle instability. Gait Posture. 2009; 29: 460-464.

6. Clark RA, Bryant AL, Pua Y, McCrory P, Bennell K, Hunt M. Validity and reliability of the Nintendo Wii Balance Board for assessment of standing balance. Gait Posture. 2010; 31: 307-310.

7. Ruhe A, Fejer R, Walker B. The test-retest reliability of centre of pressure measures in bipedal static task conditions - A systematic review of the literature. Gait Posture. 2010; 32: 436-445.

8. Clark RA, McGough R, Paterson K. Reliability of an inexpensive and portable dynamic weight bearing asymmetry assessment system incorporating dual Nintendo Wii Balance Boards. Gait Posture. 2011; 34: 288-291.

9. Yohannan SK, Tufaro PA, Hunter H, Orleman L, Palmatier S, Sang C, et al. The Utilization of Nintendo (R) Wii (TM) During Burn Rehabilitation: A Pilot Study. J Burn Care Res. 2012; 33: 36-45.

10. Hopkins S, Smith C, Toms A, Brown M, Welsman J, Knapp K. Evaluation of a dual-scales method to measure weight-bearing through the legs, and effects of weightbearing inequalities on hip bone mineral density and leg lean tissue mass. J Rehabil Med. 2013; 45: 206-210.

11. Shih $\mathrm{CH}$, Shih $\mathrm{CT}$, Chu CL. Assisting people with multiple disabilities actively correct abnormal standing posture with a Nintendo Wii Balance Board through controlling environmental stimulation. Res Dev Disabil. 2010; 31: 936-942.

12. Shih $\mathrm{CH}$, Shih $\mathrm{CT}$, Chiang MS. A new standing posture detector to enable people with multiple disabilities to control environmental stimulation by changing their standing posture through a commercial Wii Balance Board. Res Dev Disabil. 2010; 31: 281-286.

13. Shih $\mathrm{CH}$, Chang ML. A wireless object location detector enabling people with developmental disabilities to control environmental stimulation through simple occupational activities with Nintendo Wii Balance Boards. Res Dev Disabil. 2012; 33: 983-989.

14. Park D-S, Lee G. Validity and reliability of balance assessment software using the Nintendo Wii balance board: usability and validation. J Neuroeng Rehabil. 2014; 11: 99.

15. Fung V, Ho A, Shaffer J, Chung E, Gomez M. Use of Nintendo Wii FitTM In the rehabilitation of outpatients following total knee replacement: A preliminary randomised controlled trial. Physiother (United Kingdom). 2012; 98: 183-188.

16. Halton J. Virtual rehabilitation with video games: A new frontier for occupational therapy. Occup. Ther. Now. 2008; 10: 12-14.

17. Rand D, Kizony R, Weiss PTL. The Sony PlayStation II Eye Toy: low-cost virtual reality for use in rehabilitation. J Neurol Phys Ther. 2008; 32: 155-163.

18. Anderson F, Annett M, Bischof WF. Lean on Wii: Physical rehabilitation with virtual reality Wii peripherals. Stud Health Technol Inform. 2010; 154: 229234.

19. Park J-H, Lee S-H, Ko D-S. The Effects of the Nintendo Wii Exercise Program on Chronic Work-related Low Back Pain in Industrial Workers. J Phys Ther Sci. 2013; 25: 985-988.

20. Franchignoni F, Tesio L, Martino MT, Ricupero C. Reliability fo four simple, quantitative tests of balance and mobility in healthy elderly females. Aging Clin Exp Res. 1998; 10: 26-31.

21. Springer BA, Marin R, Cyhan T, Roberts H, Gill NW. Normative Values for the Unipedal Stance Test with Eyes Open and Closed. J Geriatr Phys Ther. 2007; 30: 8-15.

22. Deans SM. Determining the validity of the Nintendo Wii balance board as an assessment tool for balance [Dissertation]. University of Nevada, 2011.

23. Holmes JD, Jenkins ME, Johnson AM, Hunt MA, Clark RA. Validity of the Nintendo Wii(R) balance board for the assessment of standing balance in Parkinson's disease. Clin Rehabil. 2012; 27: 361-366.

24. Huurnink A, Fransz DP, Kingma I, van Dieën JH. Comparison of a laboratory grade force platform with a Nintendo Wii Balance Board on measurement of postural control in single-leg stance balance tasks. J Biomech. 2013; 46: 1392-1395.

25. Larsen L, Jørgensen M, Junge T, Juul-Kristensen B, Wedderkopp N. Field assessment of balance in 10 to 14 year old children, reproducibility and validity of the Nintendo Wii board. BMC Pediatr. 2014; 14: 144.

26. Sgrò F, Monteleone G, Pavone M, Lipoma M. Validity Analysis of Wii Balance Board Versus Baropodometer Platform Using an Open Custom Integrated Application. AASRI Procedia. 2014; 8: 22-29.

27. Abujaber S, Gillispie G, Marmon A, Zeni J. Validity of the Nintendo Wii Balance Board to assess weight bearing asymmetry during sit-to-stand and return-to-sit task. Gait Posture. 2015; 41: 676-682.

28. Bartlett HL, Ting LH, Bingham JT. Accuracy of force and center of pressure measures of the Wii Balance Board. Gait Posture. 2014; 39: 224-228.

29. Jorgensen MG, Laessoe U, Hendriksen C, Nielsen OBF, Aagaard P. Intrarater Reproducibility and Validity of Nintendo Wii Balance Testing in CommunityDwelling Older Adults. J Aging Phys Act. 2014; 22: 269-275. 